\title{
Impact of the New WHO Guidelines on Diagnosis and Practice of Male Infertility
}

\author{
Sandro C. Esteves ${ }^{*}, 1$ and Ashok Agarwal ${ }^{2}$ \\ ${ }^{I}$ ANDROFERT, Andrology and Human Reproduction Clinic, Campinas, São Paulo, Brazil \\ ${ }^{2}$ Center for Reproductive Medicine, Cleveland Clinic, Cleveland, Ohio, USA
}

\begin{abstract}
The World Health Organization (WHO) has established new reference values for semen characteristics in its 5th edition manual which are lower than those previously reported. Several questions arise after a careful examination of the proposed new values, especially regarding the implications of these references for diagnosis and treatment of male infertility. Despite the notable advance of using controlled studies involving couples whose time to pregnancy was less than 12 months to generate the new limits, reference studies are limited with regard to the population analyzed and the methods used for semen evaluation. As such, it seems unreasonable to assume that reference values represent global semen characteristics of fertile men as proposed in the $5^{\text {th }}$ edition WHO manual. Caution should be exercised to not overinterpret the new reference values as they may fail to accurately discriminate populations of fertile and infertile men. Properly performed semen analyses coupled with an adequate examination of the man can give valuable information related to the organs producing "semen", a highly complex fluid, and thus help in better understanding of the physiology of the reproductive organs and the causes of their dysfunctions. The present commentary discusses concerns related to the publication of the new reference values for semen parameters such as the impact on patient referral, diagnosis, treatment of recognized conditions such as varicocele and indications of assisted reproductive modalities. We conclude that more debate is needed before the adoption of the proposed WHO current reference values by andrology laboratories around the world. For those considering to adopt them, a better approach would be the presentation of reference values by percentiles rather than solely the lower cutoff limits. The time has come for technological developments that bring robust and costeffective clinically useful sperm function tests to replace, at least partially, the shortcomings of routine semen analysis.
\end{abstract}

Keywords: Male infertility, semen analysis, diagnosis, treatment, spermatozoa, opinion.

\section{INTRODUCTION}

Semen analysis is of great value in the initial investigation of male and its results are often taken as a surrogate measure of male fecundity and pregnancy risk. It provides information on the functional status of the germ epithelium, epididymis and accessory sex glands. Reference ranges for semen parameters from a fertile population may provide data from which prognosis of fertility or diagnosis of infertility can be extrapolated. Nonetheless, the prognostic value of semen components such as sperm count, motility and morphology, as surrogate markers of male fertility, is confounded in several ways; the fertility potential of a man is influenced by sexual activity, function of accessory sex glands and other conditions. Routine semen analysis itself has its own limitations, and does not assess for sperm dysfunctions such as immature chromatin or a fragmented DNA. Results from at least two, preferable three, separate seminal analyses must be obtained before a definitive conclusion can be drawn as wide biological variability exists within the same individual. Routine seminal analysis should include: a) physical characteristics of semen, including liquefaction, viscosity, $\mathrm{pH}$, color and odor; b) specimen

*Address correspondence to this author at the Av. Dr. Heitor Penteado, 1464, 13075-460, Campinas, São Paulo, Brazil; Tel: +55 19 3295-8877; Fax:+55 19 3294-6992; E-mail: s.esteves@androfert.com.br volume; c) sperm count; d) sperm motility and progression; e) sperm morphology; f) leukocyte quantification; and g) fructose detection in cases where no spermatozoa is found and ejaculate volume is low.

\section{WHAT IS NEW IN THE $5^{\text {TH }}$ EDITION WHO GUIDELINES}

The World Health Organization (WHO) periodically releases manuals for laboratory examination of human semen. The first one was published in 1980, with subsequent updates in 1987, 1992 and 1999 [1-3]. These manuals are used as a source of standard methodology for laboratories performing semen analyses worldwide. The WHO published its updated $5^{\text {th }}$ edition in late 2010 with important differences from previous versions [4]. The new edition contains more detailed information on how to analyze semen samples in a routine basis and how to perform advanced sperm function tests. It includes new chapters on sperm preparation techniques for assisted conception and cryopreservation. There is a completely revised chapter on quality control and, for the first time, multi-country data from recent fathers with known time-to-pregnancy (TTP) was incorporated.

The evidence-based reference ranges and reference limits for various semen characteristics are, in our opinion, the most important albeit controversial feature of the new manual. Reference values were obtained from data involving 
Table 1. Cut-off Values for Semen Parameters as Published in Consecutive WHO Manuals

\begin{tabular}{|c|c|c|c|c|c|}
\hline Semen parameters & WHO 1980 & WHO 1987 & WHO 1992 & WHO 1999 & WHO $2010^{1}$ \\
\hline Volume (mL) & -- & $\geq 2$ & $\geq 2$ & $\geq 2$ & 1.5 \\
\hline Sperm concentration $\left(10^{6} / \mathrm{mL}\right)$ & $20-200$ & $\geq 20$ & $\geq 20$ & $\geq 20$ & 15 \\
\hline Total sperm concentration $\left(10^{6}\right)$ & -- & $\geq 40$ & $\geq 40$ & $\geq 40$ & 39 \\
\hline Total motility (\% motile) & $\geq 60$ & $\geq 50$ & $\geq 50$ & $\geq 50$ & 40 \\
\hline Progressive motility ${ }^{2}$ & $\geq 2^{3}$ & $\geq 25 \%$ & $\geq 25 \%$ (grade a) & $\geq 25 \%$ (grade a) & $32 \%(a+b)$ \\
\hline Vitality (\% alive) & -- & $\geq 50$ & $\geq 75$ & $\geq 75$ & 58 \\
\hline Morphology (\% normal) & 80.5 & $\geq 50$ & $\geq 30^{4}$ & $(14)^{5}$ & $4^{6}$ \\
\hline Leukocyte count $\left(10^{6} / \mathrm{mL}\right)$ & $<4.7$ & $<1.0$ & $<1.0$ & $<1.0$ & $<1.0$ \\
\hline
\end{tabular}

${ }^{1}$ Lower reference limit obtained from the lower fifth centile value; ${ }^{2}$ Grade a $=$ rapid progressive motility $(>25 \mu \mathrm{m} / \mathrm{s}) ;$ grade $\mathrm{b}=\mathrm{slow} / \mathrm{sluggish}$ progressive motility $(5-25 \mu \mathrm{m} / \mathrm{s}) ;$ Normal $=50 \%$ motility (grades a + b) or $25 \%$ progressive motility (grade a) within 60 min of ejaculation; ${ }^{3}$ Forward progression (scale $0-3$ ); ${ }^{4}$ Arbitrary value; ${ }^{5}$ Value not defined but strict criterion is suggested; ${ }^{6}$ Strict (Tygerberg) criterion.

1,953 semen samples from five studies in eight countries on three continents $[5,6-10]$. Only subjects with a TTP of $\leq 12$ months were included. Semen analysis results from this group of men were pooled and analyzed to provide reference distributions for semen parameters. The mean $( \pm \mathrm{SD})$ male age was $31( \pm 5)$ years (range 18-53) and only 10 men aged more than 45 years. Laboratories generating the data used standardized methods for semen analysis according to the WHO manual for the examination of human semen which were available at the time of the original studies. In addition, data that were combined to calculate the reference distributions were provided by laboratories that practiced internal and external quality control [5]. One-sided lower reference limits (the fifth centile) were generated and were proposed as the lower cutoff limits for normality. Apart from total sperm count per ejaculate, the lower limits of these distributions are lower than the previously presented 'normal' or 'reference' values (Table 1) [1-3].

Data on normal sperm morphology, extracted from 4 studies including approximately $1,800 \mathrm{men}$, were reported according to the 'strict' (Tygerberg) method [5, 6, 8, 10, 11]. Assessment of progressive motility according to grades, as recommended by the previous WHO manuals, was replaced by categorizing motile sperm as being 'progressive' or 'nonprogressive'. This simplification in motility assessment according to the $5^{\text {th }}$ edition should allow a more objective evaluation due to the fact that sperm velocity is often interpreted subjectively by technicians. In fact, according to our own experience clinicians tend to overestimate the importance of the absence/low percentage of sperm exhibiting grade 'a' motility. This simple yet important modification will allow clinicians to focus on the proportion of progressive motile sperm rather than the type of progressive motility being rapid or slow. Sperm vitality data, assessed by the eosin-nigrosin method, was obtained from approximately 400 men of two countries [5]. Leukocyte reference values remained the same as previous manuals.

\section{WHERE THE DEBATE STARTS}

For the first time, the inclusion of reference values for semen analysis was based on controlled studies involving fertile fathers with a known TTP. As such, it represents one of the most important features of the $5^{\text {th }}$ WHO manual. In comparison, previous versions reported reference values based on the clinical experience of investigators who have studied populations of healthy fertile men of unknown TTP [1-3]. Previous WHO manuals acknowledge the limitations of their reference values by stating that each laboratory should determine its own reference range for each variable.

Despite the complex relationship between semen analysis results and pregnancy outcome, the reference values included in the current version of the WHO manual are aimed to provide evidence-based thresholds that may aid clinicians in calculating the relative fertility of a given patient. However, several concerns arise from a detailed examination of the studies which generated the current reference values [5, 6-13]. First, it should be noted that apart from a single Australian study all others came from countries situated in the northern hemisphere. The Australian study included 206 subjects which represented only about $10 \%$ of the 'fertile' reference population [6]. Roughly 55\% of the data came from four western European cities (Paris, Turku, Edinburgh and Copenhagen) and we speculate that the studies from Slama et al. [7] and Jensen et al. [9] used the same database. The remaining patients came from a small study from another western European city (Oslo) [10] and from the USA [8]. A systematic review of the literature was not performed to identify all data on semen quality in various populations. According to the authors of the original study that referenced the $5^{\text {th }}$ edition WHO manual, laboratories and data were identified through the known literature and personal communication with investigators and the editorial group of the fifth edition of the WHO laboratory manual [5]. Interestingly, four out of five studies were from the same group of authors or collaborative work among them (Table 2). Semen analyses results for the group of fertile men differed among these 'reference' studies. It was not clear if these differences represented real biological differences among men in different regions or laboratory-dependent biases of measurement, despite their adherence to the WHO manual methods. Cooper et al. [5] stated in their original report that 'the studies included in the present analysis were conducted in different regions of the world with some areas over-represented, such as Northern Europe, and others, such as Africa, parts of Europe and Central and South America, under-represented'. The point is that their reference limits 
Table 2. Characteristics of the Studies Utilized to Provide Data to Define the Reference Limits for the $5^{\text {th }}$ Edition WHO Laboratory Manual for the Examination and Processing of Human Semen

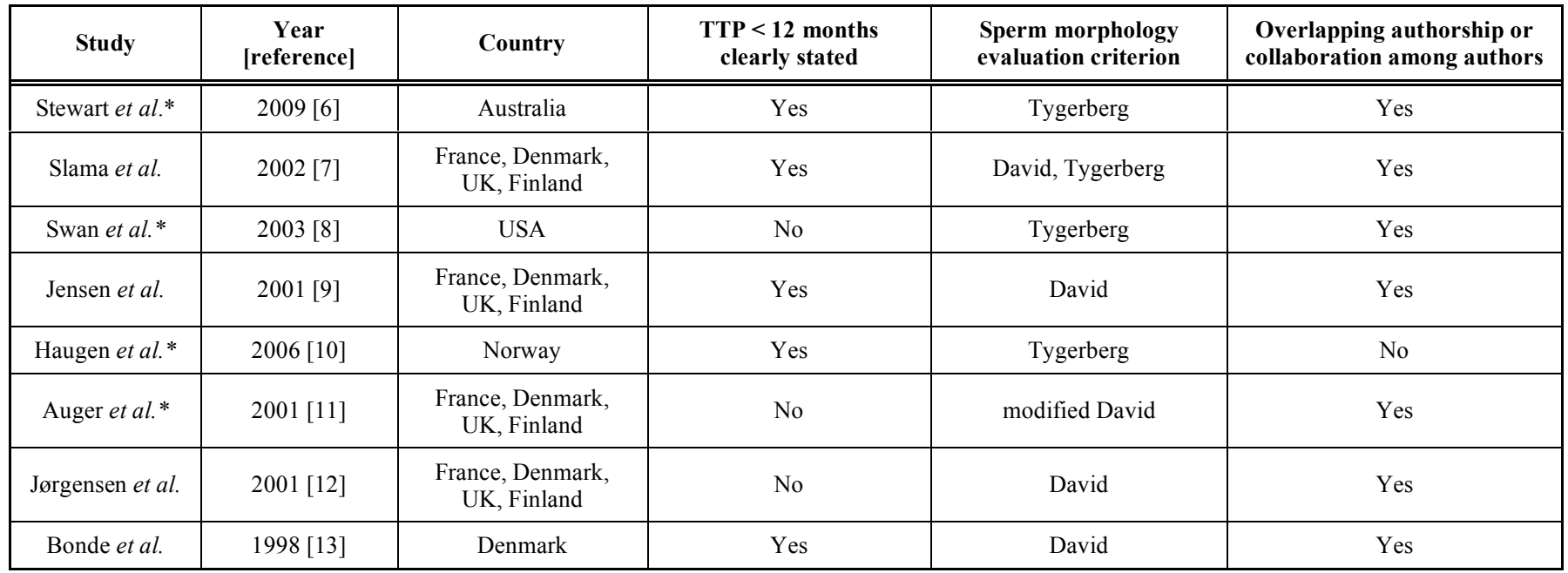

TTP $=$ Time to pregnancy

UK = United Kingdom

*Studies contributing to data on sperm morphology.

for the fertile population with known TTP came only from Northern Europe, Australia and USA; as such, other areas were not represented at all. What about millions of fertile men living in China, India, Africa, Middle East and South America? These are the areas where the vast majority of people live nowadays. From these data, it seems unsound to assume, as proposed by Cooper et al. [5], that the reference values represented global semen characteristics of fertile men.

Second, strict criterion was not the method used for assessing sperm morphology in one of the studies that had been claimed to source the reference values. Auger et al. (2001) [11] used the method originally described by David et al. (1975) [14], which differs from the one proposed by Kruger and colleagues [15]. Moreover, it should be noted that in the study by Slama et al. (2002), which sourced $46 \%$ of data, sperm morphology was also evaluated by the David's method with proper quality control. Then, morphology slides were sent to another laboratory for additional assessment of morphology according to the strict criterion by a single physician; unfortunately, no details of quality control were provided for such analysis [7].

Third, it is not easy for the reader to understand how the data from the five reference studies were pooled by Cooper and colleagues. For instance, when referring to the study by Swan et al., 593 samples were tabulated but only 512 were reported in their original study [8]. Moreover, in only two studies $[7,10]$ a TTP of $\leq 12$ months was clearly defined as an eligibility criterion for patient inclusion while in all remaining ones it has to be inferred $[6,8,9]$. To explain these discrepancies, it is likely that the original datasets were provided by the authors of these reference studies to the ones conducting the WHO study, and then the information was reorganized and reanalyzed. Lastly, a single semen sample was taken to represent each man in the reference studies. The assumption that one ejaculate is representative of a given man semen profile argues against the current knowledge of the high biological variability of semen parameters from same individuals. Several guidelines, including the WHO ones, recommend that two, but preferable three semen samples should be obtained before one man's fertility status is depicted [1-4]. It is also unlikely that a manuscript that includes a single semen sample from each individual would be considered for publication in a high-quality peer-reviewed journal in the present days; surprisingly, it was the case with the $5^{\text {th }}$ edition WHO manual.

Further studies will be required to confirm the validity of global reference ranges as proposed by the $5^{\text {th }}$ edition WHO manual. If regional differences are revealed, their mechanism and significance for fertility will need to be studied before it can be decided whether there should be specific reference values for different ethnic groups or regions. Laboratories may have to produce their own local reference ranges for semen parameters. A confirmatory analysis including a systematic review of laboratories using highly standardized techniques (such as those presented in the $5^{\text {th }}$ edition of the WHO laboratory manual) reporting participation in quality control programs, and taking geographical and ethnic origins into account, is needed. It will be of interest to determine the success of various clinical management protocols that incorporate the reference limits into research and practice guidelines.

\section{IMPLICATIONS OF THE $5^{\text {TH }}$ EDITION WHO REFERENCE VALUES FOR DIAGNOSIS AND PRACTICE}

\section{Is Male Fertility Declining?}

Reference values for semen parameters are lower in the current edition of the WHO manual compared to previous ones (Table 1) [1-4]. At a first superficial analysis, it may be concluded that the reason for such observation is a trend towards male fertility decline, as suggested by Carlsen et al. (1992) [16]. The authors' findings, suggesting that endocrine disruptors and other environmental polluters such as insecticides and pesticides are responsible for declining overall male fertility, have attracted supporters [17-20] as 
well as critics [21-25]. However, there are other reasons that may explain the difference in the reference values between the current and previous WHO manuals. One is the adherence by many laboratories of higher quality control standards especially when assessing sperm morphology. Another one, not well explored by recent commentaries, is that previous WHO reference values were mainly based on the clinical experience of investigators who have studied populations of healthy fertile men of unknown TTP rather than controlled populations of fertile men as in the current edition [1-4]. For these reasons one must exercise caution when concluding that the newly proposed lowered WHO reference values can be justified by the suggested decline in global male fertility. It may be possible that such differences are not related to the decline in male fertility at all, but rather a methodological bias created by different ways of generating data.

\section{Will Referrals for Assessment of the Male Partner Decrease?}

The answer for this question is not straightforward because it will depend on the acceptability of the new WHO manual reference values. If they are accepted by most laboratories performing semen analysis, it is likely that several patients previously categorized as having abnormal semen analysis will be now considered 'normal', and referral for evaluation may be postponed or not undertaken. It poses a potential problem since it has been exhaustively reported that male and female reproductive age are clearly associated with reproductive outcome. On the other hand, it is important to acknowledge the limitations of semen analysis results to assess the health and functional capacity of the male reproductive organs and cells. The male evaluation regarding fertility must go far beyond counting spermatozoa and assessing motility and morphology. It has to be supplemented with a proper clinical examination, a comprehensive history taking, and relevant endocrine, genetic, and/or other investigations.

\section{Are Previously Diagnosed Subfertile Men Fertile now?}

This question can also be posed as follows: "Did we overtreat our male patients before?" According to the new reference values, a man with $6 \%$ strict morphology, 16 million sperm per $\mathrm{mL}$ and $40 \%$ progressive motility is considered within the so-called 'normal' reference limits; however, the same patient is categorized as having an abnormal analysis according to the reference values proposed by the 1999 WHO manual which is still largely used [3]. According to preliminary results of a current study involving over 1,000 individuals seeking fertility evaluation, $38.7 \%$ (380/982) of the group previously classified as having abnormal semen analyzes by the WHO $4^{\text {th }}$ edition (1999) guidelines are now within the normal range (SCE; unpublished data). Do we have to correct our semen analyses reports from the previous years or call our patients for a 'recall'? Caution should be undertaken when interpreting these new reference values since it is obvious that the prevalence of couples facing difficulties to conceive has not changed because of the publication of new reference values. Values should be interpreted along with the clinical information. Every couple attempting to conceive for more than 1 year of unprotected intercourse, or less in the cases of advanced female age or in men with a recognized fertility threat, deserve medical evaluation that must include both partners irrespective of the semen analysis results. It is known that about $30 \%$ of men misdiagnosed as having unexplained male infertility, according to the normal semen parameters on routine analyses, present sperm deficiencies that can be identified by sperm function tests, such as the assessment of DNA integrity, oxidative stress and antisperm antibodies [26, 27]. Sperm DNA fragmentation and elevated oxidative stress, for instance, are recognized as having great importance in males experiencing difficulties to conceive. Abnormalities in the male genome characterized by damaged sperm DNA may be indicative of male subfertility regardless of routine semen parameters which do not reveal DNA defects. An abnormal proportion of spermatozoa with fragmented DNA can be found in 5-10\% of infertile men with normal semen analyses but is rarely seen in fertile individuals [28]. Advanced paternal age, inadequate diet intake, drug abuse, pesticide environmental exposure, tobacco use, varicocele, medical disease, scrotal hyperthermia, air pollution, genital inflammation or infectious diseases can be cited as possible causes, some of which are reversible [29]. DNA fragmentation can be secondary to internal factors such as apoptosis and oxidative stress, or external factors such as the presence of leukocytes. The oxidative stress-induced sperm damage has been suggested to be a significant contributing factor in $30-80 \%$ of all cases of male infertility [27]. Semen analyses results, when routinely performed, is limited in its validity as surrogate for male fertility potential. The couples' chances to conceive involve multiple factors and our goals, as treating physicians, are manifold. It is our responsibility to diagnose existing conditions that may compromise, now or in the future, the fertility potential of our patients, to identify potentially life-threatening diseases and to treat reversible conditions such as inadequate lifestyle habits, subclinical infections, hormone disorders and clinical varicocele.

\section{The Dilemma of Clinical Varicocele and 'Normal' Semen Parameters: To Treat, or Not to Treat? That is the Question}

Approximately $8 \%$ of men in reproductive age seek medical assistance for fertility-related problems. Of these, 1$10 \%$ carries conditions that compromise the reproductive potential, and varicocele accounts for $35 \%$ of the cases [30]. In a group of 2,875 infertile couples attending a tertiary center for male reproduction in Brazil, clinical varicoceles were identified in $21.9 \%$ of the male partners. Several studies have demonstrated that surgical treatment of clinical varicoceles is highly effective to decrease seminal oxidative stress, to increase seminal concentrations of antioxidants and to improve sperm quality [31-35]. The most recent metaanalysis on varicocelectomy by Marmar et al. (2007) unequivocally demonstrated the benefit of the surgical treatment of clinical varicoceles in infertile men with abnormal semen analyses [36]. The authors showed that the chances of spontaneous conception were 2.8 times higher in the varicocelectomy group as compared to the group of patients who received either no treatment or medication. 
The problem is that several guidelines propose that varicoceles should be treated if palpable and in the presence of abnormal semen analyses [37-39]. According to the new WHO reference semen values several patients will be ineligible for treatment when the new guidelines are strictly followed. Health insurance companies may not grant authorization or refuse reimbursement if treatment is performed in men with normal semen parameters. The question whether or not a man with clinical varicocele should undergo repair in the face of normal semen parameters according to the 5th edition WHO manual is not simply answered. What we really want is to know what would be the semen parameters of the same individual if varicocele had been treated. It would be very informative to reanalyze the meta-analysis studies on varicocelectomy to determine the magnitude of sperm quality improvement in the subgroup of patients that is now classified as having 'normal' semen. This information will certainly come, but for the time being emerging evidence seems to support the indication of treatment for men with clinical varicocele and so-called 'normal' semen parameters according to the new WHO reference values. In one study, Agarwal et al. examined the effect of varicocelectomy on semen parameters of adults and demonstrated a significant increase in sperm concentration, motility and morphology by 9.7 million $/ \mathrm{mL}$, $9.9 \%$ and $3.1 \%$, respectively [33]. In another study, Mori et al. examined a group of 360 non-selected adolescents aged 14-18 years attending a public school in Brazil [40]. They found that $27.8 \%$ presented with a palpable grade II or III varicocele but only half of them had testicular asymmetry. More importantly, semen analysis results revealed that adolescents without varicocele ejaculated significantly higher number of progressively motile sperm (134.1 million) compared to adolescents with grade II (72.7 million) and III (30.3 million) varicocele. Despite the marked difference in the seminal profile between adolescents with and without varicocele all individuals were still within the reference range for normality according to the $4^{\text {th }}$ edition WHO manual. Because semen samples of this group of adolescents are still considered normal, and because testicular asymmetry will not be present in $\geq 50 \%$ of adolescents, treatment is not recommended according to current professional societies' guidelines, such as the ones published by the American Urological Association, American Society for Reproductive Medicine, European Association of Urology and Brazilian Society of Urology [37-39]. Thus, surgical correction of the varicocele will only be offered when adolescents have already crossed into the infertile range, even though initial evaluation had already shown that their seminal profile were significantly lower than their counterparts without varicocele. These data take us back to our original argument on what would be the seminal profile of these individuals if varicocele had been treated. Due to the progressive effect of varicocele [41, 42], it is expected that treatment of varicocele halts deterioration of sperm quality and prevents individuals with yet 'normal' semen analysis to cross into the defined infertile range. Moreover, it is also possible that improvements in sperm quality after varicocele repair would increase the male reproductive potential, albeit pre- and posttreatment values are within the newly proposed reference values. Adolescents and adults with palpable varicoceles may present with normal semen analysis but altered sperm function, as shown by elevated DNA fragmentation rates and oxidative stress levels [31, 34, 43]. Taking together, this knowledge challenges the current recommendations for varicocele treatment, and highlights the importance of a continuous debate.

\section{Impact on ART Treatment: a Perfect Time for Sperm Functional Markers}

The adoption of the newly proposed WHO reference values may speed up the research and clinical application of novel sperm function tests, including the 'omics' technology [44]. The diagnosis of male subfertility will then be more precise by using novel tests of sperm function, thus decreasing the importance of routine semen analysis results. Sperm function tests may also aid in the indication of the best assisted reproductive technology (ART) modality. In clinical practice, ART, especially intracytoplasmic sperm injection (ICSI), is indicated according to the semen analysis results. It is unlikely that the new reference semen values will change the clinicians practice, at least towards the use of more complex ART such as ICSI. If a reproductive center utilizes the cutoff value of $4 \%$ strict morphology for recommending ICSI, it should not be changed because of the new guidelines since sufficient data exists to support the superiority of this technique as compared to conventional in vitro fertilization (IVF) or intrauterine insemination (IUI) in such cases [45]. Moreover, the indication of ICSI based on very poor sperm count and motility is unlikely to be changed since the thresholds will certainly be within the abnormal ranges according to the new reference values. A potential problem may arise for IUI, since several cases of mild abnormal semen analysis (that will now fit within normal reference limits) are treated by this ART modality. However, the impact of the new reference values on IUI is likely to be minimal because IUI is routinely performed in cases of unexplained infertility and normal semen analyzes. The choice of ART should be based on the clinical features of each case as well as on the Center's experience and reported results with different ART modalities rather than on semen analyses reports. It is unlikely that a clinician will modify a treatment strategy based only on the new reference values for semen parameters being considered 'normal' or 'abnormal' if his/her results with a less complex technique are poor in a particular semen scenario. It is important to stress that the reference semen values, as proposed by the WHO, cannot be over-interpreted to indicate a treatment modality. It merely represents the distribution of the semen profile of a small group of fertile individuals.

\section{Interpreting the Reference Values: Focus on the 50th Percentile}

The $5^{\text {th }}$ edition WHO manual presents semen characteristics of a population of men who had fathered a child within one year of trying to induce conception. The $95 \%$ reference intervals for a range of semen parameters and the lower reference limits have been generated in line with clinical chemistry standards $[4,5]$. For a conventional onesided distribution, the $5^{\text {th }}$ centile was proposed for the lower limit of semen characteristics. The distribution of data according to percentiles for various semen characteristics is presented in Table 3. Clinical reference values are important 
Table 3. Distribution of Values According to the 5\%, $50 \%$ and $\mathbf{9 5 \%}$ Percentiles for Semen Parameters from Fertile Men whose Partners had a Time-toPregnancy of 12 Months or Less. (Adapted from Cooper et al. World Health Organization Reference Values for Human Semen Characteristics, Hum Reprod Update 2010; 16(3): 231-45)

\begin{tabular}{|c|c|c|c|}
\hline & \multicolumn{3}{|c|}{ Centiles } \\
\hline & $\mathbf{5 \%}$ & $\mathbf{5 0 \%}$ & $\mathbf{9 5 \%}$ \\
\hline \hline Volume $(\mathrm{mL})$ & 1.5 & 3.7 & 6.8 \\
\hline Sperm count $/ \mathrm{mL}\left(\mathrm{X} 10^{6}\right)$ & 15.0 & 73.0 & 213.0 \\
\hline Sperm count/ejaculate $\left(\mathrm{X} 10^{6}\right)$ & 39.0 & 255.0 & 802.0 \\
\hline \% Motility (total) & 40 & 61 & 78 \\
\hline \% Motility (progressive) & 32 & 55 & 72 \\
\hline \% Normal* & 4 & 15 & 44 \\
\hline \% Alive $\dagger$ & 58 & 79 & 91 \\
\hline
\end{tabular}

*according to the strict (Tygerberg) criterion

†eosin-nigrosin staining

for comparison with values obtained from the patient being assessed. The observed values may be used to aid in the clinical decision making process by comparing them with reference distributions and reference intervals. Therefore, it is important not only to compare the patient results with the lower reference limit but also with the $50^{\text {th }}$ percentile, which represents $50 \%$ of the reference population of 'fertile' men. This strategy may be more realistic and can help in understanding a patient's seminal profile in relation to the reference group. Are patients presenting with 'normal' semen analysis results close to the $5^{\text {th }}$ percentile similar to those above the $50^{\text {th }}$ one? Will clinicians have a similar approach to couples whose male partners have semen parameters within normal limits but in the lower or mid percentiles depending on female age? The matter is under debate and there are certainly multiple aspects to consider in the management of the infertile couple.

\section{SHOULD THE NEW GUIDELINES BE UNIVER- SALLY ADOPTED BY ANDROLOGY LABORATO- RIES?}

At present, the matter is unresolved and certainly more debate is needed. It would be ideal to have well-funded

\begin{tabular}{|c|c|c|c|}
\hline Name: $\mathbf{X X X X X X X}$ & Age: 34 & ANDRO-086/11 & $\begin{array}{c}\text { Date: } \\
01 / 02 / 2011\end{array}$ \\
\hline \multicolumn{4}{|l|}{ Physician: Dr. Sandro Esteves } \\
\hline \multicolumn{4}{|l|}{ Reason: Initial assessment for infertility } \\
\hline Ejaculate: (X) Total & ( ) Partial & & \\
\hline Collection method: (X) Masturbation & ( ) Other: & & \\
\hline Site for collection: $\quad(X)$ Andrology lab & ( ) Home & & \\
\hline
\end{tabular}

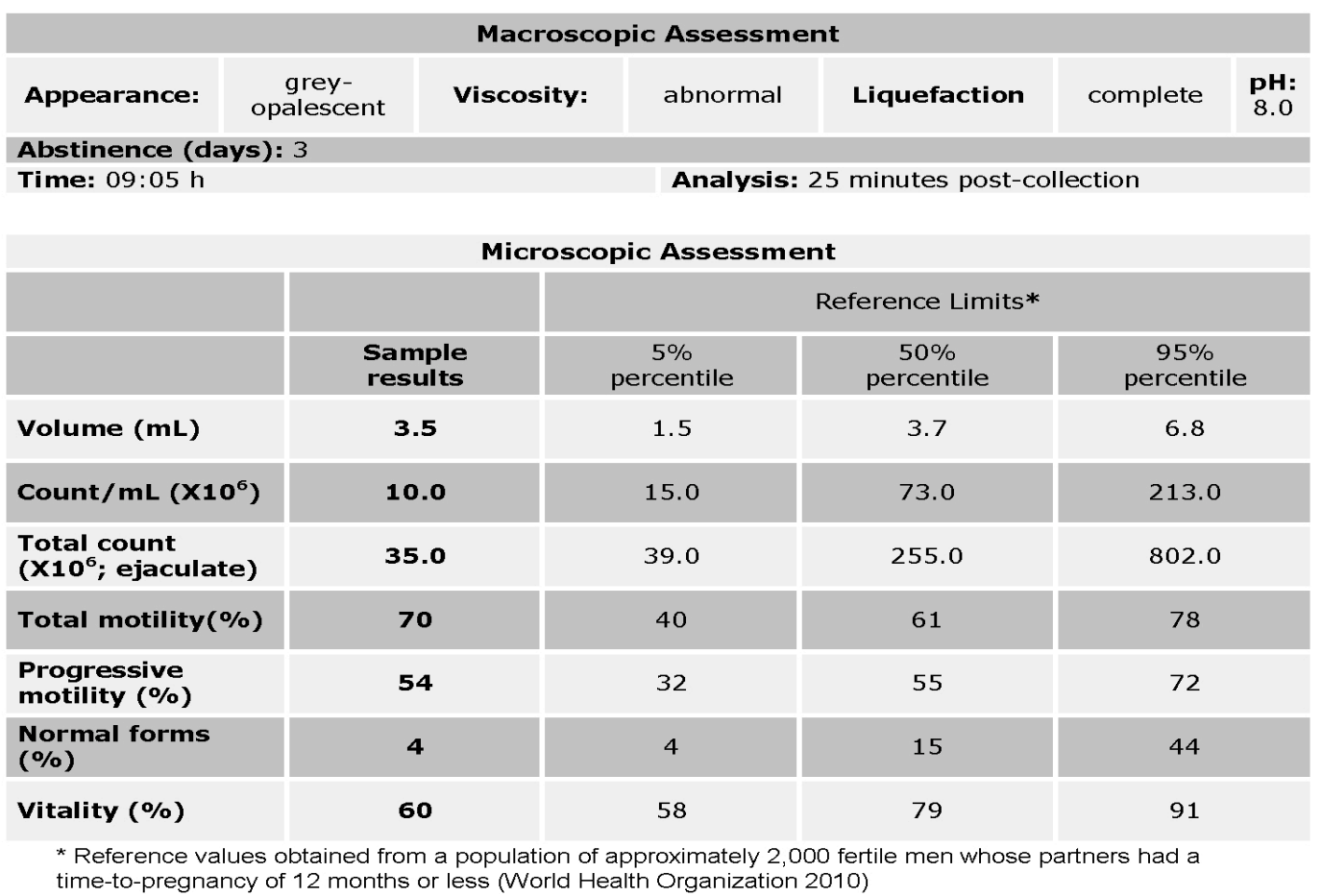

Fig. (1). Semen analysis report showing the parameters obtained from one patient (left column, as sample results) and the reference limits distributed by percentiles according to the $5^{\text {th }}$ edition WHO Laboratory Manual for the Examination and Processing of Human Semen. 
prospective studies designed to evaluate several populations of fertile men to account for geographic and racial diversity. Our andrology laboratory in Brazil decided to adopt the new WHO reference semen values. However, we elected to modify our semen analysis report form by including not only the lower reference limits but also the $50^{\text {th }}$ and $95^{\text {th }}$ percentiles (Fig. 1). We believe that the presentation of reference values according to percentiles may aid the clinician who receives the semen analysis report to better understand where his/her patient fits in comparison to the reference population. Additionally, we decided to provide extra support for clinicians requesting semen analysis, including a clear explanation that the new reference limits are derived from semen samples from men whose partners conceived spontaneously and that such reference provide only a guide regarding a patient's fertility. We further go on by explaining that semen characteristics are highly variable within and among men and these parameters are not unique determinants of a couple's ability to conceive. In other words, semen parameters within the $95 \%$ reference interval do not guarantee fertility nor do values outside those limits necessarily indicate male infertility. It is also clear from the reported reference values that $5 \%$ of the fertile men providing the reference data had values below the $5 \%$ centile defined as the minimum threshold. A man's semen characteristics need to be interpreted in conjunction with his clinical information and the reference limits should not be used to determine the nature of that treatment.

\section{KEY ISSUES}

- World Health Organization established new reference values for human semen characteristics. Men whose partners had a time-to-pregnancy of $\leq 12$ months were chosen as individuals to provide reference distributions for semen parameters, as follows: semen volume of $1.5 \mathrm{~mL}$; sperm count of 39 and 15 million per ejaculate and per $\mathrm{mL}$, respectively; vitality of $58 \%$ alive; progressive motility of $32 \%$ and morphologically normal forms (strict criteria) of $4.0 \%$. Semen parameters above the lower reference limits do not guarantee fertility nor do values outside those limits necessarily imply male infertility or pathology.

- $\quad$ Despite the notable advance of using controlled studies involving couples whose time to pregnancy was less than 12 months to generate the new limits, reference studies are limited in the population analyzed and the methods for semen evaluation. As such, it is unsound to assume, as proposed by the $5^{\text {th }}$ edition WHO manual, that the reference values represented global semen characteristics of fertile men.

- The lower reference limits for semen parameters compared to previous ones may not be related to a suggested global decline in male fertility but rather to the adherence by many laboratories of higher quality control standards, especially when assessing sperm morphology, and to the fact that previous WHO reference values were mainly obtained from the clinical experience of investigators who have studied populations of healthy fertile men of unknown TTP rather than controlled populations of fertile men as in the current edition.

- Universal acceptability of the new WHO manual reference values will classify patients previously categorized as having abnormal semen analysis as 'normal'; as such, referral for medical evaluation may be delayed. The male evaluation regarding fertility must go far beyond counting spermatozoa and assessing motility and morphology and should take into account proper clinical examination, a comprehensive history taking, and relevant endocrine, genetic, and/or other investigations.

- The prevalence of couples facing difficulties to conceive is not changed despite the publication of new reference values. Every couple attempting to conceive for more than 1 year of unprotected intercourse, or less in the cases of advanced female age or in men with a recognized fertility threat, deserve medical evaluation that must include both partners irrespective of the semen analysis characteristics. About $30 \%$ of men misdiagnosed as having unexplained male infertility, according to the normal semen parameters on routine analyses, present sperm deficiencies that are identified by sperm function tests, such as the assessment of DNA integrity, oxidative stress, antisperm antibodies, etc.

- $\quad$ Adolescents and adults with clinical varicocele may present with normal results on routine semen analysis and abnormal sperm function due sperm DNA fragmentation or elevated oxidative stress. Decreased semen quality is observed in men with clinical varicocele compared to the counterparts without varicocele, even though they may be within 'normal' limits of semen analysis according to the WHO guidelines. Current guidelines that recommend varicocele treatment based on the presence of a palpable varicocele and abnormal semen analysis should be revised.

- It is unlikely that the new WHO reference values will change clinicians' ways of utilizing ART. The choice of ART should be based on the clinical features of each case as well as on the ART center experience and reported results with different ART modalities rather than solely on the results of semen analysis.

- $\quad$ The $5^{\text {th }}$ edition WHO manual presents semen characteristics of a population of men who had fathered a child within 1 year of trying to induce a pregnancy. The $95 \%$ reference intervals for a range of semen parameters and the lower reference limits have been generated in line with clinical chemistry standards. It is important not only to compare the patient results with the lower reference limit but also with the 50th percentile, which aggregates $50 \%$ of the reference population of 'fertile' men.

- It is still uncertain whether or not the new proposed WHO reference semen values will be universally adopted by laboratories performing semen analysis. Irrespective of the reference values, a man's semen characteristics need to be interpreted in conjunction 
with his clinical information. The reference limits provided by the WHO manual are from semen samples initiating natural conceptions; as such, they may indicate the need for infertility treatment but should not be used to determine the nature of that treatment.

\section{ACKNOWLEDGEMENTS}

None Declared.

\section{CONFLICT OF INTEREST}

None Declared.

\section{REFERENCES}

[1] World Health Organization. WHO Laboratory Manual for the Examination of Human Semen and Sperm-cervical Mucus Interaction, $2^{\text {nd }}$ ed. Cambridge: Cambridge University Press 1998.

[2] World Health Organization. WHO Laboratory Manual for the Examination of Human Semen and Sperm-cervical Mucus Interaction, $3^{\text {rd }}$ ed. Cambridge: Cambridge University Press 1992.

[3] World Health Organization. WHO Laboratory Manual for the Examination of Human Semen and Sperm-cervical Mucus Interaction, $4^{\text {th }}$ ed. Cambridge: Cambridge University Press 1999.

[4] World Health Organization. WHO Laboratory Manual for the Examination and Processing of Human Semen, $5^{\text {th }}$ ed. Geneva: WHO Press 2010.

[5] Cooper TG, Noonan E, von Eckardstein S, et al. World Health Organization reference values for human semen characteristics. Hum Reprod Update 2010; 16(3): 231-45.

[6] Stewart TM, Liu DY, Garrett C, Jørgensen N, Brown EH, Baker HWG. Associations between andrological measures, hormones and semen quality in fertile Australian men: inverse relationship between obesity and sperm output. Hum Reprod 2009; 24(7): 15618.

[7] Slama R, Eustache F, Ducot B, et al. Time to pregnancy and semen parameters: a cross-sectional study among fertile couples from four European cities. Hum Reprod 2002; 17(2): 503-15.

[8] Swan SH, Brazil C, Drobnis EZ, et al. Geographic differences in semen quality of fertile U.S. males. Environ Health Perspect 2003; 111(4): 414-20.

[9] Jensen TK, Slama R, Ducot B, et al. Regional differences in waiting time to pregnancy among fertile couples from four European cities. Hum Reprod 2001; 16(12): 2697-704.

[10] Haugen TB, Egeland T, Magnus O. Semen parameters in Norwegian fertile men. J Androl 2006; 27(1): 66-71.

[11] Auger J, Eustache F, Andersen AG, et al. Sperm morphological defects related to environment, lifestyle and medical history of 1001 male partners of pregnant women from four European cities. Hum Reprod 2001; 16(12): 2710-7.

[12] Jørgensen N, Andersen AG, Eustache F, et al. Regional differences in semen quality in Europe. Hum Reprod 2001; 16(5): 1012-9.

[13] Bonde JP, Ernst E, Jensen TK, et al. Relation between semen quality and fertility: a population-based study of 430 firstpregnancy planners. Lancet 1998; 352(9122): 1172-7.

[14] David G, Bisson JP, Czyglik F. Anomalies morphologiques du spermatozoïde humain. 1. Prognitionen pour un système de classification. J Gynecol Obstet Biol Reprod 1975; 4(S1), 17-36.

[15] Kruger TF, Acosta AA, Simmons KF, Swanson RJ, Matta JF, Oehninger S. Predictive value of abnormal sperm morphology in vitro fertilization. Fertil Steril 1988; 49(1): 112-7.

[16] Carlsen E, Giwercman A, Keiding N, Skakkebaek NE. Evidence for decreasing quality of semen during past 50 years. BMJ 1992; 305(6854): 609-13.

[17] Ginsburg J, Okolo S, Prelevic G, Hardiman P. Residence in the London area and sperm density. Lancet 1994; 343(8891): 230.
[18] Auger J, Kunstmann JM, Czyglik F, Jouannet P. Decline in semen quality among fertile men in Paris during past 20 years. N Engl J Med 1995; 332(5): 281-5.

[19] Adamopoulos DA, Pappa A, Nicopoulou S, et al. Seminal volume and total sperm number trends in men attending subfertility clinics in the greater Athens area during the period 1977-1993. Hum Reprod 1996; 11(9): 1936-41.

[20] Irvine DS, Twigg JP, Gordon EL, Fulton N, Milne PA, Aitken RJ. DNA integrity in human spermatozoa: relationships with semen quality. J Androl 21(1): 33-44.

[21] Younglai EV, Collins JA, Foster WG. Canadian semen quality: an analysis of sperm density among eleven academic fertility centers. Fertil Steril 1998; 70(1): 76-80.

[22] Andolz P, Bielsa M, Villa J. Evolution of semen quality in northeastern Spain: a study in 22759 infertile men over a 36 year period. Hum Reprod 1999; 14(3): 731-5.

[23] Auger J, Jouannet P. Evidence for regional differences of semen quality among fertile French men. Hum Reprod 1997; 12(4): 740-5.

[24] Handelsman DJ. Estrogens and falling sperm counts. Reprod Fertil Dev 2001; 13(4): 317-24.

[25] Sadeu JC, Hughes CL, Agarwal S, Foster WG. Alcohol, drugs, caffeine, tobacco, and environmental contaminant exposure: Reproductive health consequences and clinical application. Clinical Rev Toxicol 2010; 40(7): 633-52.

[26] Bungum M, Bungum L, Giwercman A. Sperm chromatin structure assay (SCSA): a tool in diagnosis and treatment of infertility. AJA 2011; 13(1): 69-75.

[27] Agarwal A, Makker K, Sharma R. Clinical relevance of oxidative stress in male factor infertility: an update. Am J Reprod Immunol 2008; 59(1): 2-11.

[28] Shefi S, Turek PJ. Definition and current evaluation of subfertile men. Int Braz J Urol 2006; 32(4): 385-97.

[29] Kefer JC, Agarwal A, Sabanegh E. Role of antioxidants in the treatment of male infertility. Int J Urol 2009; 16(5): 449-57.

[30] Vital and Health Statistics, series 23, no. 26, CDC. Available from: http://www.cdc.gov. [cited: $10^{\text {th }}$ Dec 2009].

[31] Zini A, Blumenfeld A, Libman J, Willis J. Beneficial effect of microsurgical varicocelectomy on human sperm DNA integrity. Hum Reprod 2005; 20(4): 1018-21.

[32] Esteves SC, Glina S. Recovery of spermatogenesis after microsurgical subinguinal varicocele repair in azoospermic men based on testicular histology. Int Braz J Urol 2005; 31(6): 541-8.

[33] Agarwal A, Deepinder F, Cocuzza M, et al. Efficacy of varicocelectomy in improving semen parameters: new metaanalytical approach. Urology 2007; 70(3): 532-8.

[34] Smit M, Romijn JC, Wildhagen MF, et al. Decreased sperm DNA fragmentation after surgical varicocelectomy is associated with increased pregnancy rate. J Urol 2010; 183(1): 270-4.

[35] Esteves SC, Oliveira FV, Bertolla RP. Clinical outcome of intracytoplasmic sperm injection in infertile men with treated and untreated clinical varicocele. J Urol 2010; 184(4): 1442-6.

[36] Marmar JL, Agarwal A, Prabaskan S, et al. Reassessing the value of varicocelectomy as a treatment for male subfertility with a new meta-analysis. Fertil Steril 2007; 88(3): 639-48.

[37] The Male Infertility Best Practice Policy Committee of the American Urological Association; Practice Committee of the American Society for Reproductive Medicine. Report on varicocele and infertility. Fertil Steril 2004; 82(Suppl): 142-5.

[38] European Association of Urology. Guidelines on Male Infertility 2010; Available from: http://www.uroweb.org/gls/pdf/Male\% 20Infertility\%202010.pdf (cited: February $27^{\text {th }}, 2011$ ).

[39] Sociedade Brasileira de Urologia \& Colégio Brasileiro de Radiologia; Projeto Diretrizes da Associação Médica Brasileira. Varicocele. Available from: http://www.projetodiretrizes.org.br/ 8_volume/40-Varicocele.pdf (cited: February $27^{\text {th }}, 2011$ ).

[40] Mori MM, Bertolla RP, Fraietta R, Ortiz V, Cedenho AP. Does varicocele grade determine extent of alteration to spermatogenesis in adolescents. Fertil Steril 2008; 90(5): 1769-73.

[41] Gorelick JI, Goldstein M. Loss of fertility in men with varicocele. Fertil Steril 1993; 59(3): 613-6. 
[42] Witt MA, Lipshultz LI. Varicocele: a progressive or static lesion? Urology 1993; 42(5): 541-3.

[43] Bertolla RP, Cedenho AP, Hassun Filho PA, Lima SB, Ortiz V, Srougi M. Sperm nuclear DNA fragmentation in adolescents with varicocele. Fertil Steril 2006; 85(3): 625-8.
[44] Baker MA. The 'omics revolution and our understanding of sperm cell biology. AJA 2011; 13: 6-10.

[45] Coetzee K, Kruger TF, Lombard CJ. Predictive value of normal sperm morphology: a structured literature review Hum Reprod Update 1998, 4(1): 73-82.

Received: January 02, 2010

Revised: March 08, 2010

Accepted: July 26, 2010

(C) Esteves and Agarwal; Licensee Bentham Open.

This is an open access article licensed under the terms of the Creative Commons Attribution Non-Commercial License (http://creativecommons.org/licenses/by$\mathrm{nc} / 3.0 /$ ), which permits unrestricted, non-commercial use, distribution and reproduction in any medium, provided the work is properly cited. 